\title{
On Characterizations of Four Recently Introduced Distributions: Two Continuous and Two Discrete
}

\author{
G. G. Hamedani \\ Correspondence: Department of Mathematics, Statistics and Computer Science Marquette University Milwaukee, WI \\ 53201-1881. E-mail: g.hamedani@mu.edu
}

Received: May 5, 2019 Accepted: May 30, 2019 Online Published: June 13, 2019

doi:10.5539/ijsp.v8n4p25

URL: https://doi.org/10.5539/ijsp.v8n4p25

\begin{abstract}
Oluyede et al. (2016) and Mdlongwa et al. (2017) consider the continuous univariate distributions called Dagum-Poisson (DP) and Burr XII Modified Weibull (BXIIMW), respectively, and study certain properties and applications of these distributions. Shahid and Raheel (2019) and Para and Jan (2019) proposed the univariate discrete distributions called Discrete Modified Inverse Rayleigh (DMIR) and Discrete Generalized Inverse Weibull (DGIW) and study some of their mathematical properties. The present short note is intended to

complete, in some way, the works cited above via establishing certain characterizations of these distributions in different directions.
\end{abstract}

\section{Introduction}

Characterizations of distributions is an important research area which has recently attracted the attention of many researchers. This short note deals with various characterizations of DP, BXIIMW, DMIR and DGIW distributions to complete, in some way, the works cited above. These characterizations are based on: (i) a simple relationship between two truncated moments; (ii) the hazard function and (iii) conditional expectation of a function of the random variable, for DP and BXIIMW. It should be mentioned that for characterization (i) the cdf (cumulative distribution function) is not required to have a closed form. As for the DMIR and DGIW, certain characterizations of these distributions are presented based on: different form of (iii) which we call it (iii') and (iv) the reverse hazard function. Oluyede et al. (2016) introduced the DP distribution with cdf and pdf (probability density function) given, respectively, by

$$
F(x ; \lambda, \gamma, \beta, \theta)=\frac{1-\exp \left(\theta\left[1+\lambda x^{-\gamma}\right]^{-\beta}\right)}{1-e^{\theta}}, \quad x \geq 0,
$$

and

$$
f(x ; \lambda, \gamma, \beta, \theta)=\frac{\beta \lambda \gamma \theta x^{-\gamma-1}\left(1+\lambda x^{-\gamma}\right)^{-\beta-1} \exp \left(\theta\left[1+\lambda x^{-\gamma}\right]^{-\beta}\right)}{e^{\theta}-1}, \quad x>0,
$$

where $\lambda, \gamma, \beta, \theta$ are all positive parameters.

Mdlongwa et al. (2017) proposed the BXIIMW distribution with cdf, pdf and hazard function given, respectively, by

$$
\begin{gathered}
F(x ; c, k, \alpha, \beta, \lambda)=1-\left(1+x^{c}\right)^{-k} e^{-\alpha x^{\beta} e^{\lambda x}}, \quad x \geq 0, \\
f(x ; c, k, \alpha, \beta, \lambda)=\left(1+x^{c}\right)^{-k-1} e^{-\alpha x^{\beta} e^{\lambda x}}\left\{k c x^{c-1}+\left(1+x^{c}\right) \alpha x^{\beta-1} e^{\lambda x}(\beta+\lambda x)\right\}, \quad x>0,
\end{gathered}
$$

and

$$
h_{F}(x ; c, k, \alpha, \beta, \lambda)=\frac{k c x^{c-1}+\left(1+x^{c}\right) \alpha x^{\beta-1} e^{\lambda x}(\beta+\lambda x)}{\left(1+x^{c}\right)}, \quad x>0,
$$

where $c, k, \alpha, \beta$ all positive and $\lambda \geq 0$ are parameters. 
Shahid and Raheel (2019) introduced the DMIR distribution with cdf, pmf (probability mass function) and reverse hazard function given, respectively, by

$$
\begin{array}{cc}
F(x ; \alpha, \theta)=e^{-\frac{\alpha}{x}-\theta\left(\frac{1}{x}\right)^{2}}, & x \in \mathbb{N}, \\
f(x ; \alpha, \theta)=e^{-\frac{\alpha}{x}-\theta\left(\frac{1}{x}\right)^{2}}-e^{-\frac{\alpha}{x-1}-\theta\left(\frac{1}{x-1}\right)^{2}}, & x \in \mathbb{N},
\end{array}
$$

and

$$
r_{F}(x ; \alpha, \theta)=1-\frac{e^{-\frac{\alpha}{x-1}-\theta\left(\frac{1}{x-1}\right)^{2}}}{e^{-\frac{\alpha}{x}-\theta\left(\frac{1}{x}\right)^{2}}}, \quad x \in \mathbb{N},
$$

where $\alpha, \theta$ are positive parameters and $\mathbb{N}$ is the set of all positive integers.

Remark 1: Shahid and Raheel's definition of pmf results in $f(0 ; \alpha, \theta)=e^{-\alpha-\theta}$, but $F(0 ; \alpha, \theta)=0$. The corrected versions are given above.

Para and Jan (2019) proposed the DGIW distribution with cdf, pmf and reverse hazard function given, respectively, by

$$
\begin{gathered}
F(x ; \alpha, \beta)=q^{\left(\frac{\beta}{x+1}\right)^{\alpha}}, \quad x \in \mathbb{N}^{*}=\mathbb{N} \cup\{0\}, \\
f(x ; \alpha, \beta)=q^{\left(\frac{\beta}{x+1}\right)^{\alpha}}-q^{\left(\frac{\beta}{x}\right)^{\alpha}},
\end{gathered}
$$

and

$$
r_{F}(x, \alpha, \beta)=1-\frac{q^{\left(\frac{\beta}{x}\right)^{\alpha}}}{q^{\left(\frac{\beta}{x+1}\right)^{\alpha}}}, \quad x \in \mathbb{N}
$$

where $\alpha, \beta$ are positive parameters.

\section{Characterizations of DP and BXIIMW Distributions}

We present our characterizations (i)-(iii) in three subsections.

\subsection{Characterizations Based on Two Truncated Moments}

In this subsection we present characterizations of DP and BXIIMW distributions in terms of a simple relationship between two truncated moments. The first characterization result employs a theorem due to Glänzel (1987); see Theorem 2.1.1 below. Note that the result holds also when the interval $\mathrm{H}$ is not closed. Moreover, as mentioned above, it could be also applied when the cdf F does not have a closed form. As shown in Glänzel (1990), this characterization is stable in the sense of weak convergence.

Theorem 2.1.1. Let $(\Omega, F, \boldsymbol{P})$ be a given probability space and let $H=[d$; $e]$ be an interval for some $d<e$ $(d=-\infty ; e=\infty$ might as well be allowed). Let $X: \Omega \rightarrow H$ be a continuous random variable with the distribution function $\dot{F}$ and let $g$ and $h$ be two real functions defined on $H$ such that

$$
E(g(X) \mid X \geq x)=E(h(X) \mid X \geq x) \xi(x), \quad x \in H,
$$

is defined with some real function $\xi$. Assume that $g, h \in C^{1}(H), \xi \in C^{2}(H)$ and $\mathrm{F}$ is twice continuously differentiable and strictly monotone function on the set H. Finally, assume that the equation $\xi h=g$ has no real solution in the interior of $\mathrm{H}$. Then $\mathrm{F}$ is uniquely determined by the functions $g, h$ and $\xi$, particularly

$$
F(x)=\int_{a}^{x} C\left|\frac{\xi^{\prime}(u)}{\xi(u) h(u)-g(u)}\right| \exp (-s(u)) d u,
$$


where the function $s(u)$ is a solution of the differential equation $s^{\prime}=\frac{\xi^{\prime} h}{\xi h-g}$ and $C$ is the normalization constant, such that $\int_{H} d F=1$.

Proposition 2.1.1. Let $X: \Omega \rightarrow(0, \infty)$ be a continuous random variable and let $h(x)=\exp \left(-\theta\left[1+\lambda x^{-\gamma}\right]^{-\beta}\right)$ and $g(x)=h(x)\left[1+\lambda x^{-\gamma}\right]^{-\beta}$ for $x>0$. The random variable $X$ has pdf (2) if and only if the function $\xi$ defined in Theorem 2.1.1 is of the form

$$
\xi(x)=\frac{1}{2}\left\{1+\left(1+\lambda x^{-\gamma}\right)^{-\beta}\right\}, \quad x>0 .
$$

Proof. Let $X$ be a random variable with pdf (2), then

$$
(1-F(x)) E(h(X) \mid X \geq x)=\frac{1}{e^{\theta}-1}\left\{1-\left(1+\lambda x^{-\gamma}\right)^{-\beta}\right\}, \quad x>0,
$$

and

$$
(1-F(x)) E(g(X) \mid X \geq x)=\frac{1}{2\left(e^{\theta}-1\right)}\left\{1-\left(1+\lambda x^{-\gamma}\right)^{-2 \beta}\right\}, \quad x>0
$$

and finally

$$
\xi(x) h(x)-g(x)=\frac{1}{2} h(x)\left\{1-\left(1+\lambda x^{-\gamma}\right)^{-\beta}\right\}>0, \quad \text { for } x>0 .
$$

Conversely, if $\xi$ is given as above, then

$$
s^{\prime}(x)=\frac{\xi^{\prime}(x) h(x)}{\xi(x) h(x)-g(x)}=\frac{\gamma \beta \lambda x^{-\gamma-1}\left(1+\lambda x^{-\gamma}\right)^{-\beta-1}}{1-\left(1+\lambda x^{-\gamma}\right)^{-\beta}}, \quad x>0
$$

and hence

$$
s(x)=-\log \left\{1-\left(1+\lambda x^{-\gamma}\right)^{-\beta}\right\}, \quad x>0 .
$$

Now, in view of Theorem 2.1.1, $X$ has density (2).

Corollary 2.1.1. Let $X: \Omega \rightarrow(0, \infty)$ be a continuous random variable and let $h(x)$ be as in Proposition 2.1.1. The pdf of $X$ is (2) if and only if there exist functions $g$ and $\xi$ defined in Theorem 2.1.1 satisfying the differential equation

$$
\frac{\xi^{\prime}(x) h(x)}{\xi(x) h(x)-g(x)}=\frac{\gamma \beta \lambda x^{-\gamma-1}\left(1+\lambda x^{-\gamma}\right)^{-\beta-1}}{1-\left(1+\lambda x^{-\gamma}\right)^{-\beta}}, \quad x>0 .
$$

The general solution of the differential equation in Corollary 2.1.1 is

$$
\xi(x)=\left\{1-\left(1+\lambda x^{-\gamma}\right)^{-\beta}\right\}^{-1}\left[-\int \lambda \gamma \beta x^{-\gamma-1}\left(1+\lambda x^{-\gamma}\right)^{-\beta-1}(h(x))^{-1} g(x)+D\right],
$$

where $\mathrm{D}$ is a constant. We like to point out that one set of functions satisfying the above differential equation is given in Proposition 2.1.1 with $\mathrm{D}=1 / 2$. However, it should be also noted that there are other triplets $(h, \mathrm{~g}, \xi)$ satisfying the conditions of Theorem 2.1.1.

Proposition 2.1.2. Let $\mathrm{X}: \Omega \rightarrow(0, \infty)$ be a continuous random variable and let $h(x) \equiv 1$ and $g(x)=\left(1+x^{c}\right)^{-k} e^{-\alpha x^{\beta} e^{\lambda x}}$ for $x>0$. The random variable $\mathrm{X}$ has pdf (4) if and only if the function $\xi$ defined in Theorem 2.1.1 has the form

$$
\xi(x)=\frac{1}{2}\left(1+x^{c}\right)^{-k} e^{-\alpha x^{\beta} e^{\lambda x}}, \quad x>0 .
$$

Proof. Let $X$ be a random variable with pdf (4), then

$$
(1-F(x)) E(h(X) \mid X \geq x)=\left(1+x^{c}\right)^{-k} e^{-\alpha x^{\beta} e^{\lambda x}}, \quad x>0,
$$


and

$$
(1-F(x)) E(g(X) \mid X \geq x)=\frac{1}{2}\left(1+x^{c}\right)^{-2 k} e^{-2 \alpha x^{\beta} e^{2 x}}, \quad x>0,
$$

and finally

$$
\xi(x) h(x)-g(x)=-\frac{1}{2}\left(1+x^{c}\right)^{-k} e^{-\alpha x^{\beta} e^{2 x}}<0, \quad \text { for } x>0 .
$$

Conversely, if $\xi$ is given as above, then

$$
s^{\prime}(x)=\frac{\xi^{\prime}(x) h(x)}{\xi(x) h(x)-g(x)}=\frac{k c x^{c-1}+\left(1+x^{c}\right) \alpha x^{\beta-1} e^{\lambda x}(\beta+\lambda x)}{\left(1+x^{c}\right)}, \quad x>0 .
$$

Now, in view of Theorem 2.1.1, $\mathrm{X}$ has density (4).

Corollary 2.1.2. Let X: $\Omega \rightarrow(0, \infty)$ be a continuous random variable and let $h(x)$ be as in Proposition 2.1.2. The pdf of $\mathrm{X}$ is (4) if and only if there exist functions $g$ and $\xi$ defined in Theorem 2.1.1 satisfying the differential equation

$$
\frac{\xi^{\prime}(x) h(x)}{\xi(x) h(x)-g(x)}=\frac{k c x^{c-1}+\left(1+x^{c}\right) \alpha x^{\beta-1} e^{\lambda x}(\beta+\lambda x)}{\left(1+x^{c}\right)}, \quad x>0 .
$$

The general solution of the differential equation in Corollary 2.1.2 is

$$
\xi(x)=\left(1+x^{c}\right) e^{\alpha x^{\beta} \lambda x}\left[\begin{array}{l}
-\int\left\{c k x^{c-1}+\left(1+x^{c}\right) \alpha e^{\lambda x} x^{\beta-1}(\beta+\lambda x)\right\}\left(1+x^{c}\right)^{-k-1} e^{-\alpha x^{\beta} \lambda x} \\
\times(h(x))^{-1} g(x) d x+D
\end{array}\right],
$$

where $\mathrm{D}$ is a constant. Note that a set of functions satisfying the above differential equation is given in Proposition 2.1.2 with $\mathrm{D}=0$. However, it should be also noted that there are other triplets $(h, g, \xi)$ satisfying the conditions of Theorem 2.1.1.

\subsection{Characterization Based on Hazard Function}

It is known that the hazard function, $h_{F}$, of a twice differentiable distribution function, $F$, satisfies the first order differential equation

$$
\frac{f^{\prime}(x)}{f(x)}=\frac{h_{F}^{\prime}(x)}{h_{F}(x)}-h_{F}(x) .
$$

For many univariate continuous distributions, this is the only characterization available in terms of the hazard function. The following proposition establishes a characterization of BXIIMW distribution in terms of the hazard function, which is not of the above trivial form.

Proposition 2.2.1. Let $\mathrm{X}: \Omega \rightarrow(0, \infty)$ be a continuous random variable. The pdf of $\mathrm{X}$ is (4) if and only if its hazard function $h_{F}(x)$ satisfies the differential equation

$$
h_{F}^{\prime}(x)+\frac{c x^{c-1}}{\left(1+x^{c}\right)} h_{F}(x)=\left(1+x^{c}\right)^{-1} \frac{d}{d x}\left(k c x^{c-1}+\left(1+x^{c}\right) \alpha x^{\beta-1} e^{\lambda x}(\beta+\lambda x)\right), \quad x>0,
$$

with the initial condition $h_{F}(0)=0$ for $\mathrm{c}>0$ and $\beta>0$.

Proof. If X has pdf (4), then clearly the above differential equation holds. Now, if the differential equation holds, then

$$
\frac{d}{d x}\left\{\left(1+x^{c}\right) h_{F}(x)\right\}=\frac{d}{d x}\left(k c x^{c-1}+\left(1+x^{c}\right) \alpha x^{\beta-1} e^{\lambda x}(\beta+\lambda x)\right), \quad x>0,
$$

or

$$
h_{F}(x)=\frac{k c x^{c-1}+\left(1+x^{c}\right) \alpha x^{\beta-1} e^{\lambda x}(\beta+\lambda x)}{\left(1+x^{c}\right)}, \quad x>0,
$$


which is the hazard function of the BXIIMW distribution.

\subsection{Characterizations Based on Conditional Expectation}

The following proposition has already appeared in Hamedani (2013), so we will just state it here which can be used to characterize the BXIIMW distribution.

Proposition 2.3.1. Let X: $\Omega \rightarrow(a, b)$ be a continuous random variable with cdf F. Let $\psi(x)$ be a differentiable function on $(a, b)$ with $\lim _{x \rightarrow a^{+}} \psi(x)$. Then for $\delta \neq 1$,

$$
E[\psi(X) \mid X \geq x]=\delta \psi(x), \quad x \in(a, b),
$$

if and only if

$$
\psi(x)=\{1-F(x)\}^{\frac{1}{\delta}-1}, \quad x \in(a, b) .
$$

Remark 2.3.1. For $(a, b)=(0, \infty), \psi(x)=\left(1+x^{c}\right)^{-1} e^{-\frac{\alpha}{k} x^{\beta} e^{2 x}}$ and $\delta=\frac{k}{k+1}$, Proposition 2.3 .1 provides a characterization of BXIIMW distribution. Of course there are other suitable functions than the one we mentioned above, which is chosen for simplicity.

\section{Characterizations of DMIR and DGIW Distributions}

We present our characterizations (iii') and (iv) in the following two subsections.

\subsection{Characterizations Based on Conditional Expectation}

Proposition 3.1.1. Let $X: \Omega \rightarrow \mathbb{N}$ be a random variable. The pmf of $X$ is (7) if and only if

$$
E\left\{\left(e^{-\frac{\alpha}{X}-\theta\left(\frac{1}{X}\right)^{2}}+e^{-\frac{\alpha}{X-1}-\theta\left(\frac{1}{X-1}\right)^{2}}\right) \mid X \leq k\right\}=e^{-\frac{\alpha}{k}-\theta\left(\frac{1}{k}\right)^{2}} \quad x \in \mathbb{N} .
$$

Proof. If $X$ has pmf (7), then the left-hand side of (12) will be

$$
(F(k))^{-1} \sum_{x=1}^{k}\left\{\left(e^{-2 \frac{\alpha}{x}-2 \theta\left(\frac{1}{x}\right)^{2}}-e^{-2 \frac{\alpha}{x-1}-2 \theta\left(\frac{1}{x-1}\right)^{2}}\right) \mid X \leq k\right\}=e^{-\frac{\alpha}{k}-\theta\left(\frac{1}{k}\right)^{2}} e^{-2 \frac{\alpha}{k}-2 \theta\left(\frac{1}{k}\right)^{2}}=e^{-\frac{\alpha}{k}-\theta\left(\frac{1}{k}\right)^{2}}, \quad x \in \mathbb{N} .
$$

Conversely, if (12) holds, then

$$
\sum_{x=1}^{k}\left\{\left(e^{-\frac{\alpha}{x}-\theta\left(\frac{1}{x}\right)^{2}}+e^{-\frac{\alpha}{x-1}-\theta\left(\frac{1}{x-1}\right)^{2}}\right) f(x)\right\}=F(k) e^{-\frac{\alpha}{k}-\theta\left(\frac{1}{k}\right)^{2}}
$$

From (12), we also have

$$
\sum_{x=1}^{k-1}\left\{\left(e^{-\frac{\alpha}{x}-\theta\left(\frac{1}{x}\right)^{2}}+e^{-\frac{\alpha}{x-1}-\theta\left(\frac{1}{x-1}\right)^{2}}\right) f(x)\right\}=F(k-1) e^{-\frac{\alpha}{k-1}-\theta\left(\frac{1}{k-1}\right)^{2}}=(F(k)-f(k)) e^{-\frac{\alpha}{k-1}-\theta\left(\frac{1}{k-1}\right)^{2}} .
$$

Now, subtracting (14) from (13), we arrive at

$$
f(k) e^{-\frac{\alpha}{k}-\theta\left(\frac{1}{k}\right)^{2}}=F(k)\left(e^{-\frac{\alpha}{k}-\theta\left(\frac{1}{k}\right)^{2}}-e^{-\frac{\alpha}{k-1}-\theta\left(\frac{1}{k-1}\right)^{2}}\right) .
$$

From the last equality, we have

$$
r_{F}(k)=\frac{f(k)}{F(k)}=1-\frac{e^{-\frac{\alpha}{k-1}-\theta\left(\frac{1}{k-1}\right)^{2}}}{e^{-\frac{\alpha}{k}-\theta\left(\frac{1}{k}\right)^{2}}}, \quad k \in \mathbb{N}
$$


which, in view of (8), implies that $\mathrm{X}$ has pmf (7).

Proposition 3.1.2. Let $X: \Omega \rightarrow \mathbb{N}^{*}$ be a random variable. The pmf of $X$ is (10) if and only if

$$
E\left\{\left(q^{\left(\frac{\beta}{X+1}\right)^{\alpha}}+q^{\left(\frac{\beta}{X}\right)^{\alpha}}\right) \mid X \leq k\right\}=q^{\left(\frac{\beta}{k+1}\right)^{\alpha}}-q^{-\left(\frac{\beta}{k+1}\right)^{\alpha}+2 \beta^{\alpha}}, \quad k \in \mathbb{N}^{*} .
$$

Proof. Is similar to that of Proposition 3.1.1 and hence is omitted.

\subsection{Characterization Based on Reverse Hazard Function}

Proposition 3.2.1. Let $X: \Omega \rightarrow \mathbb{N}$ be a random variable. The pmf of $X$ is (7) if and only if its reverse hazard function satisfies the difference equation

$$
r_{F}(k+1)-r_{F}(k)=\frac{e^{-\frac{\alpha}{k-1}-\theta\left(\frac{1}{k-1}\right)^{2}}}{e^{-\frac{\alpha}{k}-\theta\left(\frac{1}{k}\right)^{2}}}-\frac{e^{-\frac{\alpha}{k}-\theta\left(\frac{1}{k}\right)^{2}}}{e^{-\frac{\alpha}{k+1}-\theta\left(\frac{1}{k+1}\right)^{2}}},
$$

with the boundary condition $r_{F}(1)=1$.

Proof. If $X$ has pmf (7), then clearly (16) holds. Now if (16) holds, then for every $x \in \mathbb{N}$, we have

$$
\sum_{k=1}^{x-1}\left[r_{F}(k+1)-r_{F}(k)\right]=\sum_{k=1}^{x-1}\left\{\frac{e^{-\frac{\alpha}{k-1}-\theta\left(\frac{1}{k-1}\right)^{2}}}{e^{-\frac{\alpha}{k}-\theta\left(\frac{1}{k}\right)^{2}}}-\frac{e^{-\frac{\alpha}{k}-\theta\left(\frac{1}{k}\right)^{2}}}{e^{-\frac{\alpha}{k+1}-\theta\left(\frac{1}{k+1}\right)^{2}}}\right\},
$$

or

$$
r_{F}(x)-r_{F}(1)=\frac{e^{-\frac{\alpha}{x-1}-\theta\left(\frac{1}{x-1}\right)^{2}}}{e^{-\frac{\alpha}{x}-\theta\left(\frac{1}{x}\right)^{2}}} .
$$

In view of the fact that $r_{F}(1)=1$, from the last equation we have

$$
r_{F}(x)=1-\frac{e^{-\frac{\alpha}{x-1}-\theta\left(\frac{1}{x-1}\right)^{2}}}{e^{-\frac{\alpha}{x}-\theta\left(\frac{1}{x}\right)^{2}}}
$$

which, in view of (8), implies that $X$ has pmf (7).

Proposition 3.2.2. Let $X: \Omega \rightarrow \mathbb{N}^{*}$ be a random variable. The pmf of $X$ is (10) if and only if its reverse hazard function satisfies the difference equation

$$
r_{F}(k+1)-r_{F}(k)=\frac{q^{\left(\frac{\beta}{k}\right)^{\alpha}}}{q^{\left(\frac{\beta}{k+1}\right)^{\alpha}}}-\frac{q^{\left(\frac{\beta}{k+1}\right)^{\alpha}}}{q^{\left(\frac{\beta}{k+2}\right)^{\alpha}}}
$$

with the boundary condition $r_{F}(1)=1$.

Proof. Is similar to that of Proposition 3.2.1 and hence is omitted.

\section{References}

Glänzel, W. (1987). A characterization theorem based on truncated moments and its application to some distribution families, Mathematical Statistics and Probability Theory (Bad Tatzmannsdorf, 1986), Vol. B, Reidel, Dordrecht, 75-84. https://doi.org/10.1007/978-94-009-3965-3_8

Glänzel, W. (1990). Some consequences of a characterization theorem based on truncated moments, Statistics: A Journal of Theoretical and Applied Statistics, 21(4), 613-618. https://doi.org/10.1080/02331889008802273 
Hamedani, G. G. (2013). On certain generalized gamma convolution distributions II, Technical Report, No. 484, MSCS, Marquette University.

Mdlongwa, P., Oluyede, B. O., Amey, A., \& Huang, S. (2017). The Burr XII modified Weibull distribution: model, properties and applications, Electronic J. of Applied Statistical Analysis, 10(1), 118-145.

Oluyede, B. O., Motsewabagale, G., \& Huang, S. (2016). Warahena-Liyanage, G. and Pararai, M., The Dagum-Poisson distribution: model, properties and application, Electronic J. of Applied Statistical Analysis, 9(1), 169-197.

Para, B. A., \& Jan, T. R. (2019). On three parameters discrete generalized inverse Weibull distribution: properties and applications. AODS. https://doi.org/10.1007/s40745-018-0184-x

Shahid, N., \& Raheel, R. (2019). Discrete modified inverse Rayleigh distribution. Pak. J. Statist, 35(1), 75-95.

\section{Copyrights}

Copyright for this article is retained by the author(s), with first publication rights granted to the journal.

This is an open-access article distributed under the terms and conditions of the Creative Commons Attribution license (http://creativecommons.org/licenses/by/4.0/). 